\title{
Electromagnetic waves in layered conductors and superlattices in a magnetic field
}

\author{
V. M. Gvozdikov and R. Vega-Monroy \\ Department of Physics, Kharkov State University, 310077, Kharkov, Ukraine \\ E-mail: vladimir.m.gvozdikov@univer.kharkov.ua \\ Received January 28, 1999, revised March 12, 1999
}

\begin{abstract}
The collective electromagnetic wave excitations in layered conductors in an external quantizing magnetic field are studied theoretically. A set of coupled equations for the electric field components and the Greens functions describing collective electromagnetic modes in such a system within the linear-response approach are obtained on the basis of a model of conducting planes embedded into a dielectric matrix. These equations, written in the layer-site representation, provide a basis for studies of the electromagnetic waves in the general case which does not imply either the spatial uniformity across the layers or the homogenity within the conducting planes. The dispersion equations are obtained for the uniform and homogeneous layered conductors, which give a rich structure of collective electromagnetic modes in layered conductors in an external magnetic field. The dispersion and damping of helicons in layered conductors in the regimes of conventional and quantum Hall effects have been studied, both analytically and numerically. Two new modes propagating perpendicular to the magnetic field with frequencies of the order of the plasma frequency have been found.
\end{abstract}

PACS: 73.20.Dx, 73.20.Mf, 73.40.Hm

\section{Introduction}

It is known that weakly damped electromagnetic waves of different types can propagate in metals in a strong magnetic field at low temperatures $[1,2]$. Among them are the spiral waves, or helicons, whose frequency $\omega$ is proportional to the intensity of the magnetic field $H$, (Ref. 3) the magnetoplasma waves $[4,5]$, the Alfven waves, the cyclotron waves [6], surface waves [7], magnetoimpurity waves $[8,9]$, and other waves.

A progress gained in fabrication of various artificial superlattices as well as recent extensive research of different types of layered metallic, organic, and other (semi) conductors and superconductors, including high- $T_{c}$ cuprates, enhances activity in studies of collective electromagnetic oscillations in these systems.

A quasi-two-dimensional ( $2 D)$ nature of the electron spectrum in superlattices exerts a substantial impact on the shape of a $2 D$ conductivity tensor which, together with Maxwell's equations, determines the spectrum and the damping of the electromagnetic waves in superlattices.

The practical interest in such systems is determined by the possibility of varying the spectral properties of the electromagnetic waves in superlattices by modifying their structural parameters and varying the strength of the external magnetic field.

Extensive literature on the plasma and electromagnetic modes in different types of superlattices is available [10-21]. At the same time, the electromagnetic waves in layered conductors in an external magnetic field have been studied less extensively [22-26]. The $2 D$ dynamics of electrons in a quantizing magnetic field produces some nontrivial phenomena and concepts such as the quantum Hall effect (QHE) (Ref. 27) and anyons (Ref. 28), for example. The QHE was observed experimentally in a superlattice [29] and layered organic conductors $[30,31]$. The electromagnetic waves (helicons) in layered conductors under the conditions of the QHE have been considered in a number of theoretical studies [22,24-26] within the model of periodic array of $2 D$ electron gas embedded in a homogeneous dielectric substrate. In this model electrons have no dispersion across the layers so that the quasiclassical approach of the standard electron theory of metals based on the concept of the Fermi surface $[2,32]$ and its version adapted for the semiconducting superlattices [10] should be modified. The dispersion relation for the electromagnetic 
waves propagating along the field (i.e., across the layers) in such quasi- $2 D$ superlattices is governed by the interlayer electromagnetic correlations described by the Maxwell's equations.

The relationship between the electromagnetic field and the current within the layers is determined by the $2 D$ conductivity tensor $\sigma_{\alpha \beta}(H)$, which is the key quantity for the electromagnetic wave dispersion. The dispersion of helicons is determined by the Hall components $\sigma_{x y}=-\sigma_{y x}$, which are quantized in quasi- $2 D$ conductors under the conditions of the quantum Hall effect and by the longitudinal component $\sigma_{x x}$, which is nonzero at frequency $\omega \neq 0$ due to polarization and displacement currents $[26,33]$ in QHE. The role of the electron dispersion across the layers in high-frequency phenomena and propagation of electromagnetic waves have been considered in Ref. 34. Some specific features of helicons in layered conductors related to the quasi- $2 D$ electron energy spectrum were discussed in Ref. 35.

The purpose of this paper (which is the first publication of a series of studies on the wave propagation in layered conductors and superconductors) is twofold. First, we derive a basic system of equations describing electromagnetic field propagation in layered conductors and superlattices in the most general form, in particular, for nonuniform spatial distribution of the dielectric substance between the layers, nonperiodic layer sequence and inhomogeneities within the conducting layers. These equations provide a basis for subsequent studies of the problem in question which will be published elsewhere.

Another aim of this paper is to study further some problems, which have so far not been resolved completely, on the basis of our model in the limiting case of a uniform and homogeneous layered conductors. In particular, we consider the dispersion and damping of the helicon waves in layered conductors under conditions of conventional and quantum Hall effect and study their coupling to the intra-layer plasmons.

\section{The model and basic equations}

Consider an infinite stack of conducting planes separated by dielectric layers of thickness $a$, which is large enough so that one can ignore hopping between adjacent layers. This model is known to be a good approximation for some natural layered crystals, such as Tl- or Bi- based high- $T_{c}$ superconducting copper oxides, for example, and evidently it may be perfectly well realized in an artificially fabricated semiconductor or metallic superlattices.
In this section we consider the wave equations within the frame of the above model. To this end, we direct the $z$ axis perpendicular to the layers and assume that a constant external magnetic field $H$ is also directed along this axis. We adopt that the permeability of the substance between the layers equals to unity, $\mu=1$ and also assume its dielectric constant, $\varepsilon=\varepsilon(z)$, to be a function of $z$.

Under these assumptions, the Maxwell's equations, written in terms of the electric field $\mathbf{E}$,

$$
\operatorname{grad}(\operatorname{div} \mathbf{E})-\Delta \mathbf{E}=-\frac{\varepsilon}{c^{2}} \frac{\partial^{2} \mathbf{E}}{\partial t^{2}}+\frac{4 \pi}{c^{2}} \frac{\partial}{\partial t} \mathbf{J}
$$

after the substitution

$$
E_{l}=E_{l}(\mathbf{q}, z, \omega) \exp [i(\mathbf{q} \boldsymbol{\rho}-\omega t)], \quad(l=x, y, z),
$$

takes the form

$$
\begin{gathered}
-\mathbf{q}\left(\mathbf{q} \mathbf{E}_{\perp}\right)+i \mathbf{q}\left(\frac{\partial}{\partial z} E_{z}\right)+\left(q_{\omega}^{2}-\frac{\partial^{2}}{\partial z^{2}}\right) \mathbf{E}_{\perp}=-\frac{4 \pi i \omega}{c^{2}} \mathbf{J}_{\perp}, \\
E_{z}=-\frac{1}{q_{\omega}^{2}} \frac{\partial}{\partial z}\left(i \mathbf{q} \mathbf{E}_{\perp}\right), \\
q_{\omega}^{2}(z)=q^{2}-\frac{\omega^{2}}{c^{2}} \varepsilon(z) .
\end{gathered}
$$

Here $\boldsymbol{\rho}, \mathbf{q}$, and $\boldsymbol{\omega}$ are the in-plane coordinate, the wave-vector and the frequency of the collective mode, and $\mathbf{E}_{\perp}$ and $\mathbf{J}_{\perp}$ are the in-plane field and the current, respectively.

Choosing $\mathbf{q}$ to be parallel to $y$ axis, we obtain the system of equations

$$
\begin{gathered}
\left(\frac{\partial^{2}}{\partial z^{2}}-q_{\omega}^{2}\right) E_{x}=\frac{4 \pi i \omega}{c^{2}} J_{x} \\
\left(\frac{\partial^{2}}{\partial z^{2}}-q_{\omega}^{2}\right) E_{y}+U(q, \omega, z) \frac{\partial}{\partial z} E_{y}=-\frac{4 \pi i q_{\omega}^{2}}{\omega \varepsilon(z)} J_{y},
\end{gathered}
$$

$$
\begin{gathered}
E_{z}=-\frac{i q}{q_{\omega}^{2}} \frac{\partial}{\partial z} E_{y}, \\
U(q, \omega, z)=\left(\frac{q}{q_{\omega}(z)}\right)^{2} \varepsilon^{-1}(z) \frac{\partial \varepsilon(z)}{\partial z} .
\end{gathered}
$$

Thus, we see that all three components of the electric field are determined by the two equations (6) and (7), which can be rewritten as follows: 


$$
\begin{gathered}
E_{x}(n)=\frac{4 \pi i \omega}{c^{2}} \sum_{n^{\prime}} G_{q_{\omega}}^{x}\left(n, n^{\prime}\right)\left[\sigma_{x x} E_{x}\left(n^{\prime}\right)+\sigma_{x y} E_{y}\left(n^{\prime}\right)\right] \\
E_{y}(n)=-\frac{4 \pi i q_{\omega}^{2}}{\omega} \times \\
\times \sum_{n^{\prime}} G_{q_{\omega}}^{y}\left(n, n^{\prime}\right)\left[\sigma_{y y} E_{y}\left(n^{\prime}\right)+\sigma_{y x} E_{x}\left(n^{\prime}\right)\right] \varepsilon^{-1}(a n)
\end{gathered}
$$

We took into account here that the current can flow only within the layers and therefore its $z$ axis distribution contains a system of $\delta$-functions

$$
J_{\alpha}=\sum_{\beta, n} \sigma_{\alpha \beta}(\mathbf{q}, \omega, H) \delta(z-a n) E_{\beta}(\mathbf{q}, \omega, z) .
$$

The subscripts $\alpha$ and $\beta$ can take two values $x$ and $y$. The Green's functions in (10) $G_{q_{\omega}}^{\alpha}\left(n, n^{\prime}\right) \equiv$ $\equiv G_{q_{\omega}}^{\alpha}\left(a n, a n^{\prime}\right)$ satisfy the equations

$$
\begin{gathered}
\left(\frac{\partial^{2}}{\partial z^{2}}-q_{\omega}^{2}(z)\right) G_{q_{\omega}}^{x}\left(z, z^{\prime}\right)=\delta\left(z-z^{\prime}\right), \\
\left(\frac{\partial^{2}}{\partial z^{2}}+U(\mathbf{q}, \omega, z) \frac{\partial}{\partial z}-q_{\omega}^{2}(z)\right) G_{q_{\omega}^{y}}^{y}\left(z, z^{\prime}\right)=\delta\left(z-z^{\prime}\right) .
\end{gathered}
$$

The in-plane conductivity tensor $\sigma_{\alpha \beta}$ has off-diagonal Hall components because of the external magnetic field $H$, which we assume to be applied perpendicular to the layers.

The principal difference of our approach from the one developed in the previous papers $[22,23]$ is that we do not imply a spatial uniformity of the $\varepsilon(z)$ across the layers. We also do not imply periodicity of the layer sequence in Eq. (10) since $\varepsilon(z)$ between the layers in fact may have an arbitrary constant value. [In the case of aperiodic layer sequence the quantities $a$ and $n$ in Eq. (11) should be replaced by $a_{n}$ - the discrete coordinates of the conducting planes].

In this paper we consider the bulk modes in the case of a regular and uniform layered conductor under conditions of the conventional and quantum Hall regimes, leaving the nonuniform case for separate publication.

In the case of an infinite regular and uniform, $\varepsilon(z)=$ const, layered crystal the $U$-term in Eq. (13) vanishes and the Green's functions $G_{q}^{x}\left(z, z^{\prime}\right)$ and $G_{q}^{y}\left(z, z^{\prime}\right)$ become identical since they both satisfy Eq. (12), in which $q_{\omega}(z)$ is independent of $z$. The Green's function of Eq. (12) can then be easily found

$$
G_{q_{\omega}}\left(z, z^{\prime}\right)=-\frac{1}{2 q_{\omega}} \exp \left(-q_{\omega}\left|z-z^{\prime}\right|\right) .
$$

Making the Fourier transform

$$
E_{\beta}(n)=\frac{a}{2 \pi} \int_{-\pi / a}^{\pi / a} d k \exp (\text { ikan }) E_{\beta}(k)
$$

and completing summation over the layer index in Eqs. (10), we can rewrite them in the matrix form

$$
\sum_{\beta}\left[\delta_{\alpha \beta}-\frac{2 \pi i \omega}{c^{2} q_{\omega}} \sigma_{\alpha \beta}(q, \omega, H) V_{\alpha \beta} S(\mathbf{q}, k, \omega)\right] E_{\beta}(q, k, \omega)=0 .
$$

The structural formfactor $S(\mathbf{q}, k, \omega)$ in Eq. (16) is given by

$S(\mathbf{q}, k, \omega)=\sum_{n=-\infty}^{\infty} \mathrm{e}^{-q_{\omega} a|n|+i k n}=\frac{\sinh \left(q_{\omega} a\right)}{\cosh \left(q_{\omega} a\right)-\cos (k a)}$

and the components of the matrix $V_{\alpha \beta}$ are

$$
V_{11}=V_{12}=1, \quad V_{21}=V_{22}=-\frac{c^{2} q_{\omega}^{2}}{n_{0}^{2} \omega^{2}},
$$

where $n_{0}=\sqrt{\varepsilon}$ is the refraction index.

The system of two uniform equations has nonzero solutions if the following condition is satisfield:

$\operatorname{det}\left[\delta_{\alpha \beta}-\frac{2 \pi i \omega}{c^{2} q_{\omega}} \sigma_{\alpha \beta}(\mathbf{q}, \omega, H) V_{\alpha \beta} S(\mathbf{q}, k, \omega)\right]=0$,

which is the dispersion equation for the bulk modes which determine the frequency $\omega=\omega(\mathbf{q}, k, H)$ of a wave that can propagate in the volume of a layered conductor. The key quantity in (19) is the $2 D$ conductivity tensor $\sigma_{\alpha \beta}$, which depends on the layer structure and the external magnetic field. Thus, the specific type of the electromagnetic wave is determined by the particular form of the tensor $\sigma_{\alpha \beta}$. We consider some types of bulk waves in the following sections on the basis of the dispersion equation (19).

\section{The helicon modes}

According to the Eq. (19) the wave dispersion in layered conductors is determined by the in-plane conductivity tensor which depends on the electronic 
properties of the layer and the intensity of the external magnetic field.

The in-plane conductivity may be anisotropic without the external magnetic field as is the case, for example, in some layered organic (super) conductors $[30,31]$.

We consider here a more simple case of a two-dimensional electron gas in a perpendicular magnetic field. The conductivity tensor for this model was calculated elsewhere (see Ref. 36) and has the following components:

$$
\begin{gathered}
\sigma_{x x}=\sigma_{0} \gamma\left(1+\gamma^{2}\right)^{-1}, \quad \sigma_{x y}=-\sigma_{0}+\gamma \sigma_{x x} \\
\sigma_{x x}=\sigma_{y y}, \quad \sigma_{x y}=-\sigma_{y x}
\end{gathered}
$$

where

$$
\sigma_{0}=\frac{N e^{2}}{m \Omega}, \quad \gamma=\frac{v-i \omega}{\Omega} .
$$

Here $\Omega=e H / m c$ stands for the cyclotron frequency, $v=1 / \tau$ is the Landau level damping due to the impurities, and $N$ is the $2 D$ electron density.

Substituting Eqs. (20) and (21) into the dispersion equation (19), we have

$$
\begin{gathered}
1+\gamma^{2}-\frac{i \gamma S(\mathbf{q}, k, \omega)}{2 \Omega_{*}}\left(\frac{\omega_{*}^{2} \tilde{\omega}}{q_{\omega} a}-\frac{q_{\omega} a}{n_{0}^{2} \tilde{\omega}}\right)+ \\
+S^{2}(\mathbf{q}, k, \omega)\left(\frac{\omega_{*}}{\Omega_{*}}\right)^{2} \frac{1}{4 n_{0}^{2}}=0 .
\end{gathered}
$$

The dimensionless notations adopted in this relation are

$$
\omega_{*}=\frac{\omega_{p} a}{c}, \quad \Omega_{*}=\frac{\Omega}{\omega_{p}}, \quad \tilde{\omega}=\frac{\omega}{\omega_{p}},
$$

and $\omega_{p}^{2}=4 \pi N e^{2} / m a$ is the plasma frequency.

The helicons, as is well known, propagate along the magnetic field direction in the bulk conventional metals $[2,3]$ and in the layered conductors $[22,23]$. Thus, to study their dispersion and damping we must consider the case $q a=0$. The layer formfactor $S(\mathbf{q}, k, \omega)$ in this case takes the form

$$
S(0, k, \omega) \simeq \frac{i \omega_{*} \tilde{\omega} n_{0}}{1-\cos (k a)} .
$$

We have taken into account also that $\omega_{*} \tilde{\omega} n_{0}<<1$, (this statement will be verified below), so that we can approximate $\cosh \left(\omega_{*} \tilde{\omega} n_{0}\right) \approx 1$ in the denomina- tor of Eq. (17). Using (25), we rewrite the dispersion relation (23) in the form

$$
1+(\Gamma-i X)^{2}-i(\Gamma-i X) X 2 A-X^{2} A^{2}=0,
$$

where

$$
\Gamma=\frac{\nu}{\Omega}, \quad X=\frac{\omega}{\Omega}, \quad A=\frac{\omega_{*}^{2}}{2(1-\cos k a)} .
$$

The equation (26) has an exact solution in the complex form $X=\operatorname{Re} X-i \operatorname{Im} X$, which determines the dispersion of helicons along the magnetic field

$$
\operatorname{Re} \omega(k)=\frac{\Omega \sin ^{2}(k a / 2)}{\left(\omega_{*} / 2\right)^{2}+\sin ^{2}(k a / 2)},
$$

and their damping

$$
\operatorname{Im} \omega(k)=\frac{v \sin ^{2}(k a / 2)}{\left(\omega_{*} / 2\right)^{2}+\sin ^{2}(k a / 2)} .
$$

In the long wavelength limit, $k a \rightarrow 0$, the helicon dispersion relation (28) gives the well-known result $[22,23]$

$$
\operatorname{Re} \omega(k) \simeq \frac{\Omega}{\omega_{p}^{2}} k^{2} c^{2} .
$$

The damping of helicons, as one can see from Eq. (29), is proportional to $v$ and vanishes in a pure conductor $(v=0)$. The small quantity $\omega_{*}<<1$ may vary in a wide range, depending on the values of the plasma frequency $\omega_{p}$ and the distance between layers $a$. The appropriate dispersion $\operatorname{Re} \omega(k)$ and the damping $\operatorname{Im} \omega(k)$, considered as functions of the wave vector $k$, are shown in Fig. 1 for different values of $v$.

It follows from Eqs. (28) and (29) that the ratio

$$
\frac{\operatorname{Im} \omega(k)}{\operatorname{Re} \omega(k)}=\frac{\nu}{\Omega}
$$

is independent of the wave vector $k$, so that for $v<<\Omega$ helicons are damped only slightly at any $k a$. The above-required smallness of the parameter $\omega_{*} \tilde{\omega} n_{0}$ holds since, according to the Eq. (28), $\tilde{\omega} \sim \Omega / \omega_{p}$ and $\omega_{*} \tilde{\omega} n_{0} \sim \Omega a / c<<1$ because in attainable fields for thicknesses $a \simeq 10^{-7}-10^{-5} \mathrm{~cm}$, $\Omega$ is much lower the frequency $c / a \sim 10^{17}-10^{15} \mathrm{~s}^{-1}$.

\section{The helicon waves under the conditions of the quantum Hall effect}

The dispersion equation (19) is a general relation which may be applied to various layered conductors 

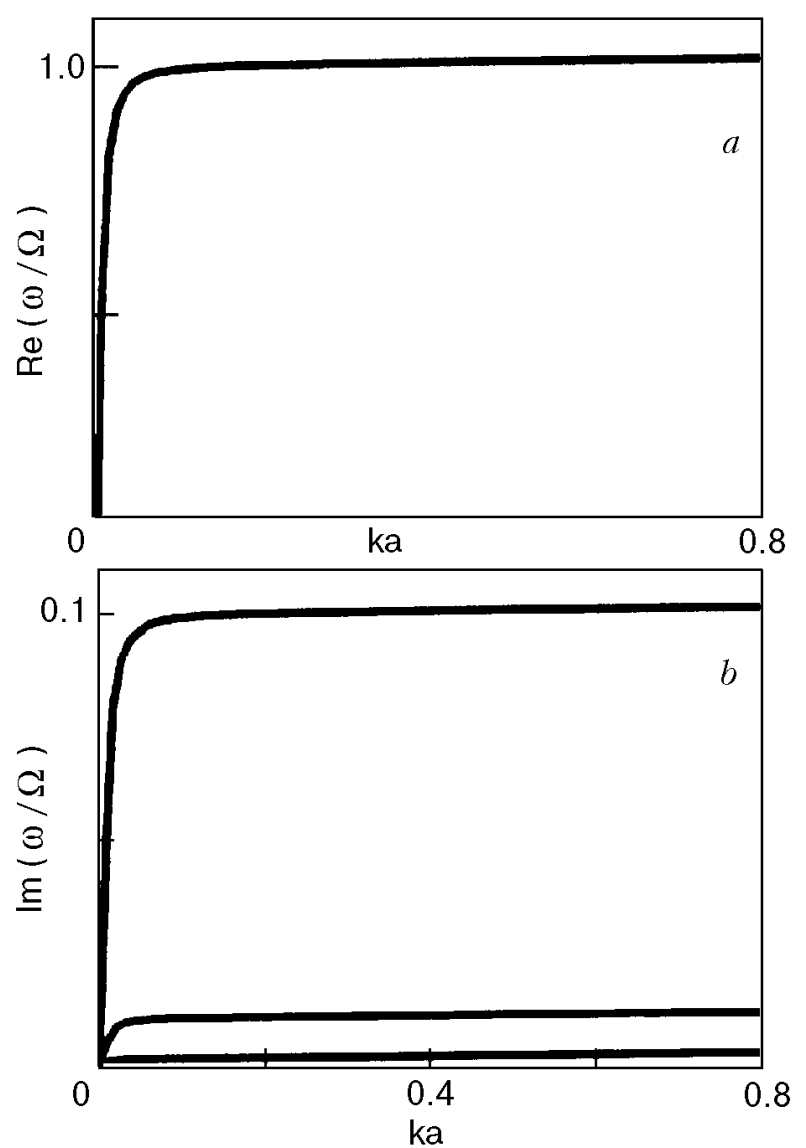

Fig. 1. The dispersion relation (a) and the damping (b) of helicons in a layered conductor under the condition the Hall effect. The parameter $\omega_{*}=0.01$ for both pictures. Re $\omega$ is independent of the Landau level broadening, whereas $\operatorname{Im} \omega$ is proportional to $v$, taken in $(b)$ equal to $0.1,0.01$, and 0.001 , respectively, from the top line to the bottom line [see also Eqs. (28) and (29)].

with different types of conductivity tensors. The helicon waves of the preceding section are caused by the Hall currents and formally stem from the Hall components of the conventional conductivity tensor $\sigma_{\alpha \beta}$ of the $2 D$ electron gas. On the other hand, $\sigma_{\alpha \beta}$ has a very simple form in a $2 D$ system under the conditions of the quantum Hall effect. In view of this circumstance, it is rather logical to consider the problem of the helicons in layered conductors under the QHE conditions on the basis of our approach, especially since QHE was observed in some layered organic conductors [30,31] and superlattices [29].

Early theoretical studies of helicons under the conditions of the QHE (Refs. 22, 24 and 25) disregarded the effect of the polarization currents on the $\sigma_{\alpha \beta}(\omega)$ at nonzero frequency [26,33], which makes the $\operatorname{Im} \sigma_{x x}(\omega) \neq 0$. The longitudinal conductivity in this case is

$$
\sigma_{x x}(\omega)=i\left(\frac{\omega}{\Omega}\right) \sigma_{x y}
$$

where $\sigma_{x y}=-\sigma_{y x}$ is the quantized, in units of the conductance quantum, $e^{2} / h$, static component of the conductivity tensor [27]

$$
\sigma_{x y}=\frac{n e^{2}}{h}, \quad(n=1,2,3, \ldots) .
$$

With respect of Eq. (33), the dispersion equation (19) gives

$$
\begin{gathered}
{\left[1+\alpha S(\mathbf{q}, k, \omega)\left(\frac{\omega}{\Omega}\right) \sigma_{x y}\right] \times} \\
\times\left[1-\beta S(\mathbf{q}, k, \omega)\left(\frac{\omega}{\Omega}\right) \sigma_{x y}\right]+\alpha \beta S^{2}(\mathbf{q}, k, \omega) \sigma_{x y}^{2}=0,
\end{gathered}
$$

where

$$
\alpha=\frac{2 \pi \omega}{c^{2} q_{\omega}} \text { and } \beta=\frac{2 \pi q_{\omega}}{\omega n_{0}} .
$$

Setting in Eq. (34) $q a=0$ and using the formfactor $S(0, \omega, k)$ in the form $(25)$, we obtain a very simple dispersion equation for helicons under the QHE regime

$$
1+2 X^{2} A_{n}-A_{n}^{2} X^{2}\left(1-X^{2}\right)=0
$$

where $X=\omega / \Omega$, and

$$
A_{n}=\frac{n \alpha_{0}}{1-\cos (k a)} \frac{\Omega a}{c}, \quad(n=1,2,3, \ldots) .
$$

Here $\alpha_{0}=c^{2} / \hbar c=1 / 137$ is the fine structure constant.

The solution of Eq. (36) is trivial and yields two modes $\omega_{+}(k a)$ and $\omega_{-}(k a)$;

$$
\left(\frac{\omega_{ \pm}}{\Omega}\right)^{2}=\frac{2-A_{n} \pm \sqrt{A_{n}\left(A_{n}-4\right)}}{2 A_{n}} \ldots
$$

For small $k a<<1$ (when $A_{n} \rightarrow 1$ ) we have the following relation for the $\omega_{-}(k a)$ mode, which corresponds to the «minus» sign in Eq. (38),

$$
\omega_{-}(k a) \simeq \frac{2 c}{a n \alpha_{0}} \sin ^{2} \frac{k a}{2} .
$$

Formally, the magnetic field does not enter Eq. (39) but, in fact, it does since (39) is valid only for fields $H$ which fall within the plateaus in the quantum Hall conductivity [27].

Another branch of the $\omega(k a)$, which corresponds to the positive sign in the dispersion relation (38), in the long-wavelength limit is given by 


$$
\omega_{+}(k a) \simeq \Omega-\frac{2 c}{a n \alpha_{0}} \sin ^{2} \frac{k a}{2} .
$$

Thus, we have two types of wave in a layered conductor in the QHE regime: one with the acoustic dispersion $\left[\omega_{-}(k a)\right]$ and one with the opticlike dispersion $\left[\omega_{+}(k a)\right]$. The $\omega_{+}(k a)$ branch has not been considered before. It appears in the problem in question owing to the term $\sigma_{x x}(\omega)$ (32) in the dispersion equation (34). This term enhances by unity the power of the polynomial in $X^{2}$ in the left-hand side of Eq. (36) and, correspondingly, gives an additional root [i.e., the new branch of $\left.\omega_{+}(k a)\right]$. Physically, the frequency-dependent longitudinal conductivity (32), as was shown in Refs. 26 and 33 , is caused by the polarization currents.

It follows from Eq. (38) that under the condition $A_{n}(k a)<4$ the right-hand side of Eq. (38) acquires an imaginary part, which implies that $\operatorname{Im} \omega(k a) \neq 0$.

An analysis shows that for $k$ less than the threshold value, $k^{*}$, the quantum waves in question propagate without damping $[\operatorname{Im} \omega(k a)=0]$. The threshold value is

$$
k^{*}(n)=\frac{2}{a} \arcsin \left(\frac{\pi n}{4 \alpha_{0}} \frac{\Omega a}{c}\right)^{1 / 2} .
$$

For $k>k^{*}$ the $\operatorname{Im} \omega_{ \pm}(k a) \neq 0$.

The dispersion and the damping of the two modes in layered conductors in the QHE regime are shown in Fig. 2 for the first three values of the integer $n=1,2,3$. We see from Fig. 2 that the decreasing modes $\omega_{+}(k a)$ do exist only for $k<k^{*}(n)$ [they terminate exactly at $k=k^{*}(n)$, as is shown in Fig. 2,a] whereas the modes increasing with $k a \omega_{-}(k a)$ are damped when $k>k^{*}(n)$, because - Im $\omega(k a) \neq 0$. This damping, as one can see in Fig. 2,c, increases rapidly with $k a$, so that the ratio $\left|\operatorname{Im} \omega_{-}(k a) / \operatorname{Re} \omega_{-}(k a)\right|<<1$ only in a small vicinity of the threshold value $k^{*}$.

\section{The bulk modes with dispersion along the layers}

The dispersion equation (23) which we have used in Sec. 3 for studies of the helicon waves that propagates along the magnetic field may be also employed for analysis of different types of waves in this model. In this section we will briefly discuss the waves that propagate perpendicular to the magnetic field, i.e., parallel to the layers, and then will consider the case $q \neq 0, k \neq 0$, both analytically and numerically.
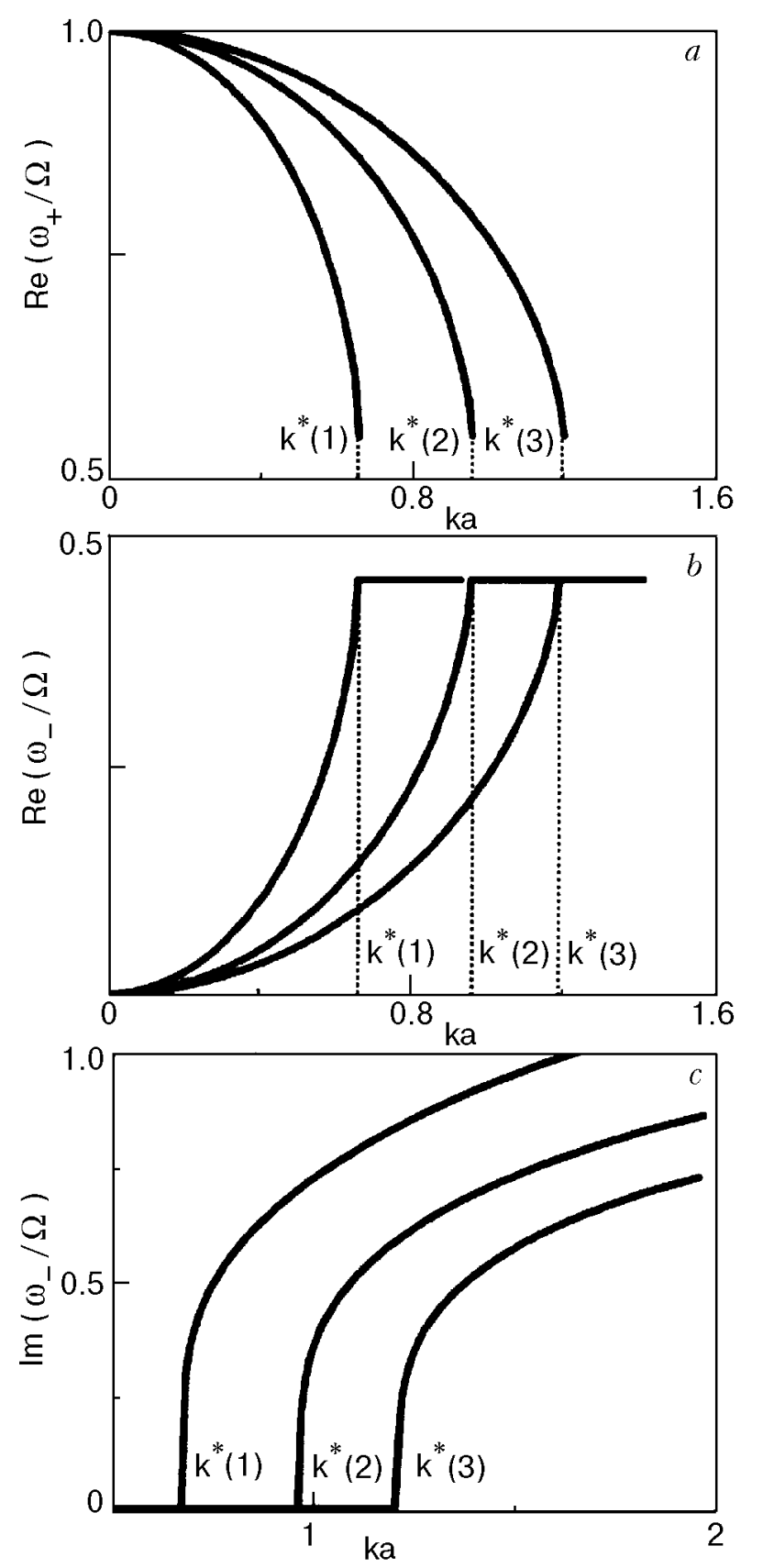

Fig. 2. The dispersion relation and the damping of helicons, given by Eq. (38), under the conditions of the QHE in a layered conductor are shown in $(a-c)$ for integers $n=1,2,3$. The decreasing modes $\omega_{+}(k)$ in $(a)$ do not exist above the threshold values $k>k^{*}(n)$ [see Eq. (4) for $k^{*}(n)$ ]. The increasing modes $\omega_{-}(k)$ in $(b)$ are damped when $k>k^{*}(n)$ since $\operatorname{Im} \omega_{-}(k) \neq 0$ in (c) under this condition.

The analytic solution of Eq. (23) can be obtained, in particular, for the case of waves propagating exactly parallel to the layers, i.e., when $k=0$, provided that $\left|q_{\omega} a\right|<<1$, where $q_{\omega}$ is given by Eq. (5). Under these conditions, setting $v=0$ and taking into account that the layer formfactor (17) in this limit is 


$$
S(\mathbf{q}, 0, \omega) \approx \operatorname{coth}\left(\frac{q_{\omega} a}{2}\right) \approx \frac{2}{q_{\omega} a},
$$

we have from Eq. (23)

$$
y^{2}-y(1+\beta)+\beta=0,
$$

where

$$
y=\left(\frac{\omega}{\omega_{p}}\right)^{2} n_{0}, \quad \beta=\frac{1}{n_{0}}\left[1+\left(\frac{q a}{\omega_{*}}\right)^{2}\right] .
$$

The solution of Eq. (43) gives two modes:

$$
\omega_{1}^{2}=\left(\frac{\omega_{p}}{n_{0}}\right)^{2}\left[1+\left(\frac{q a}{\omega_{*}}\right)^{2}\right] \text { and } \omega_{2}^{2}=\frac{\omega_{p}^{2}}{n_{0}} .
$$

The mode $\omega_{1}$ belongs to the so-called propagating sector $q_{\omega}^{2}<0$ (Ref. 23) which can be checked by the direct substitution of Eq. (45) into the definition for $q_{\omega}(6)$, which yields $q_{\omega}^{2} a^{2}=-\omega_{*}^{2}$. Thus, $\left|q_{\omega} a\right|=\left|\omega_{*}\right|<<1$, which has been assumed in the course of the derivation of Eq. (45). We also assume that $|\gamma|=|\omega / \Omega|>>1$, which is true for modes $\omega_{1}$ and $\omega_{2}$ since $\omega_{p}>>$.

The mode $\omega_{2}$ after substitution into Eq. (6) yields $q_{\omega}^{2} a^{2}=(q a)^{2}-n_{0} \omega_{*}^{2}$, so that the condition $\left|q_{\omega} a\right|<<1$ holds for $q a<<1$.

On the other hand, it is possible to obtain an analytic solution of Eq. (23) when $q a \gg>n_{0} \omega_{*} \omega / \omega_{p}$. In this case $q_{\omega} a \approx q a$, so that the formfactor of the layer is independent of $\omega$ :

$$
S(q, k, 0) \approx \frac{\sinh (q a)}{\cosh (q a)-\cos (k a)} .
$$

Under these conditions the dispersion relation (23) in the limit $v=0$ yields

$$
\omega^{2}=2 q a\left[\frac{\Omega^{2}}{2 q a+S \omega_{*}^{2}}+\left(\frac{\omega_{p}}{2 n_{0}}\right)^{2} S\right] .
$$

Setting $k a=0$ in Eq. (46), we have for small $q a$ (i.e., for $\left.n_{0} \omega_{*} \omega / \omega_{p}<q a<<1\right) \quad S(q, 0,0) \approx$ $\approx 2 / q a$, which, after substitution into the Eq. (47), yields

$$
\omega^{2} \approx \Omega^{2}+\left(\frac{\omega_{p}}{n_{0}}\right)^{2} \approx\left(\frac{\omega_{p}}{n_{0}}\right)^{2} .
$$

We see, therefore, that in this limit the dispersion relation (47) equals the $\omega_{1}$ mode, given by Eq. (45). Since $\omega_{*}=\omega_{p} a / c$ is of the order of $10^{-4}-10^{-2}$ for $\omega_{p} \stackrel{*}{\simeq} 10^{13}-10^{15}$ and $a \simeq 10^{-7}-10^{-5}$, the inequality $q a>>n_{0} \omega_{*} \omega / \omega_{p} \simeq \omega_{*}$ holds for $\omega$, given by Eq. (48).

The second term in Eq. (47) is exactly the bulk plasmon dispersion in a layered conductor [12-15]

$$
\omega_{p l}(q)=\left(\frac{\omega_{p}}{n_{0}}\right)^{2} \frac{q a}{2} \frac{\sinh (q a)}{\cosh (q a)-\cos (k a)} .
$$

The first term in Eq. (47) under the conditions $k a \neq 0, q a<<1$ may be rewritten in the heliconlike form

$$
\Omega^{2}\left[1+\frac{S \omega_{*}^{2}}{2 q a}\right]^{-1} \approx \frac{\Omega^{2} \sin ^{2}(k a / 2)}{\sin ^{2}(k a / 2)+\left(\omega_{*} / 2\right)^{2}} .
$$

We thus conclude that when the wave vector has both components, parallel (q) and perpendicular $(k)$ to the layers, the waves in question are the mixture of helicons and plasmons. For $q=0$ only helicons are excited.

The damping of the mode (47) in the linear approximation on the small parameter $v / \Omega<1$ is given by

$$
\operatorname{Im} \omega(q)=\frac{v}{2}(1+\hat{S})^{-1}\left[(2+\hat{S})\left(\frac{\omega}{\omega_{p}}\right)^{2}-\frac{q a}{n_{0}^{2}}\right],
$$

where

$$
\hat{S}=\frac{S(q, k, 0) \omega_{*}^{2}}{2 q a}
$$

and $\left(\omega / \omega_{p}\right)^{2}$ is determined by Eq. (47).

In the case $k a=0$ and for small $q a<<1$ the formfactor $S(q, 0,0) \approx 2 / q a$ and the damping is estimated to be

$$
\operatorname{Im} \omega(q) \approx \frac{v}{2}[S(q, 0,0)-1] \frac{q a}{n_{0}^{2}} \approx \frac{v}{n_{0}^{2}} .
$$

It is easy to find the dependence of helicons on $q a$ in the case of a small $q a, q a<<1$. The dispersion equation (23) in this case yields

$$
\begin{gathered}
1+(\Gamma-i X)^{2}-(X+i \Gamma) \times \\
\times A\left(2 X-\frac{\tilde{q}^{2}}{X}\right)+A^{2}\left(\tilde{q}^{2}-X^{2}\right)=0 .
\end{gathered}
$$

This equation differs from (26) in the terms containing $\tilde{q}^{2}$. The latter is defined by the relation

$$
\tilde{q}=\frac{q a}{n_{0} \omega_{*} \Omega_{*}} .
$$




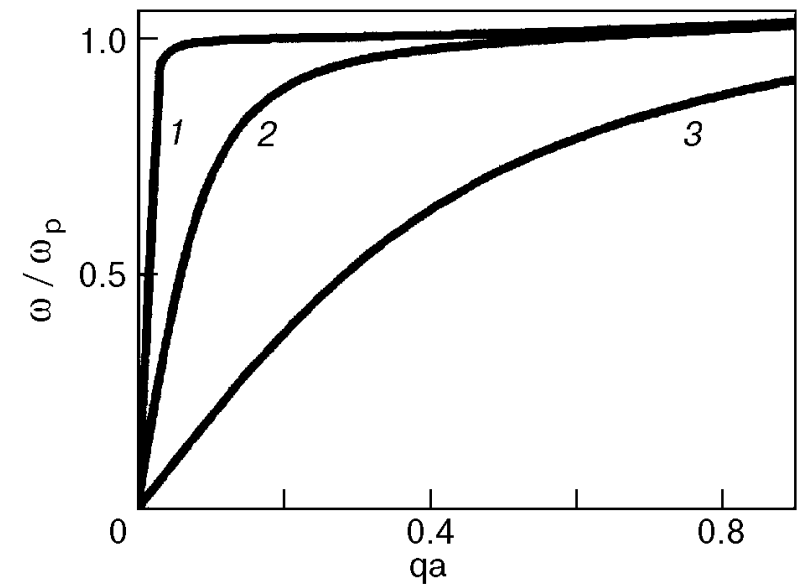

Fig. 3. The dispersion relation $\omega(q a)$ for electromagnetic waves in a layered conductor in an external magnetic field plotted as a function of the in-plane component of the wave vector $q$, calculated on the basis of Eq. (23) for three different values of the component parallel to the magnetic field: $k a=0.01$ (1); 0.1 (2); 0.5 (3). The other parameters are: $\omega_{*}=0.01$, $\Omega / \omega_{p}=0.001, v=0$, and $n_{0}=1$.

The $\tilde{q}^{2}$ terms make the dispersion relation be cubic in powers of $X$ [in constrast with the quadratic equation (23)] so that when $v \neq 0$, it can be solved only in linear on $v / \Omega<1$ approximation. The result is

$$
\begin{array}{r}
\operatorname{Re} \omega=\frac{\Omega \sin ^{2}(k a / 2)}{\left(\omega_{*} / 2\right)^{2}+\sin ^{2}(k a / 2)} \beta^{1 / 2,} \\
\operatorname{Im} \omega=\frac{v \sin ^{2}(k a / 2)}{\left(\omega_{*} / 2\right)^{2}+\sin ^{2}(k a / 2)}-\frac{v}{2 \beta} \tilde{q}^{2} A,
\end{array}
$$

where

$$
\beta=1+A(1+A) \tilde{q}^{2} .
$$

These equations give corrections to the dispersion relation and damping of helicons given by Eqs. (23) and (29) due to the small terms proportional to the parameter,

$$
\tilde{q}^{2} A=\left(\frac{q a}{n_{0} \Omega_{*} 2 \sin (k a / 2)}\right)^{2}<<1 .
$$

The numerical calculations of the dependence $\omega(q)$, obtained for different values of the parameter $k a$ both in propagating $\left(q_{\omega} a\right)^{2}<0$ and in nonpropagating $\left(q_{\omega} a\right)^{2}>0$ sectors, are shown in Fig. 3 .

\section{Summary and discussion}

We have considered the electromagnetic waves propagating in a stack of conducting layers em- bedded into a dielectric matrix in the presence of an external magnetic field perpendicular to the layers. We ignored in our model the interlayer electron hopping and do not imply either periodicity in the layer stacking or uniformity of the dielectric constant across the layers. The latter distinguishes our approach, which is based on Eqs. (10) and those for the Green's functions [Eqs. (12) and (13)], from those presented in the preceding papers on this subject $[22,23]$. As a first step toward the studies of the electromagnetic waves with the help of the equations which we obtained, we have considered the case of the bulk modes in the regular and uniform layered conductors and superlattices.

The fundamental quantity which determines the specific form of the electromagnetic wave dispersion is the conductivity tensor of a two-dimensional layer in a perpendicular magnetic field. We took $\sigma_{\alpha \beta}$ in the Drude-like form (20)-(22), which yields in the case $q a=0$ the well-known helicons that propagate along the magnetic field in layered conductors [22]. Our new finding here is the expression for damping of these helicons, given by Eq. (29). It is shown that $\operatorname{Im} \omega(k)$ is proportional to the Landau level broadening $v$ and is independent of $H$, so that the ratio (31) of the wave damping to its frequency equals $v / \Omega$ and is independent of the wave vector $k$. The dispersion and damping of helicons under the conventional Hall effect regime are shown in Fig. 1. The corrections to the dispersion relation and damping of helicons due to the deviation of the wave vector from the field direction (i.e., a small portion of the components which are parallel to the layers, $q a<<1$ ) are given by Eqs. (56) and (57).

The helicons in a highly doped InAs-GaSb artificial superlattice with a two-dimensional electron gas have been observed in Ref. 29 and it is tempting to try to search for them in the high- $T_{c}$ layered cuprates. At first sight, Tl- or Bi-based layered cuprates seem to satisfy well our model because of the high anisotropy and low conductivity across the layers in the normal state. Unfortunately, these strongly correlated systems display a very high broadening $v \simeq \omega$ (Ref. 37), which means that their damping $\operatorname{Im} \omega(k) \approx v \simeq \Omega$ is of the same order of magnitude as the wave frequency $\operatorname{Re} \omega(k) \simeq \Omega$.

Helicons in the QHE regime in superlattices have been studied extensively theoretically [2426]. The experimental grounds for this are given by the observation of QHE in some artificial superlattices [29] and layered crystals [30,31]. The QHE in organic conductors remains so far unresolved theoretically [36]. 
Of all the studies of the helicons in the QHE regime in superlattices, only the paper of Vagner and Bergman [26] takes into account that at radiofrequencies Im $\sigma_{x x}$, given by Eq. (32), is nonzero. They used a model with the Kronig-Penney-like dispersion relation for the electromagnetic waves across the layers and obtained for $k a<<1$ the result (39), which is a particular limiting case of our more general expression for the helicon dispersion at arbitrary wave vector $k a(38)$. In addition to the low-frequency mode, $\omega_{-}(k a)$, we found a high-frequency mode, $\omega_{+}(k a)(40)$ and showed that both modes can propagate without damping only if $k<k^{*}$. The threshold wave vector depends on the magnetic field $H$ and is given by Eq. (41). The dispersion and damping of helicons under the QHE regime are shown in Fig. 2.

We also have shown that the waves propagating along the layers have frequencies [given by Eq. (45)] of the order of the plasma frequency $\omega_{p}$, and we have calculated both analytically [Eqs. (47) and (48)] and numerically (see Fig. 3) their dispersion relations for different values of $k$ and $q$. For $k \neq 0$ these modes may be considered as a mixture of helicons and plasmons, since their frequency $\omega \approx \Omega$ for $q a<<1$ [see the Eqs. (49) and (50)]. The dispersion shown in Fig. 3 is qualitatively similar to that found in Ref. 39 for the coupled hole-like and electron-like two-dimensional magnetoplasmons in a semiconductor-insulator structure with metallized surfaces in the presence of a magnetic field perpendicular to the layers.

In summary, we would like to stress that there is no way to consider all the possibilities of the dispersion relations (10) and (19) in one article. In particular, we leave for the following separate publication our results obtained, together with A. M. Ermolaev on the magnetoimpurity modes in the layered conductors considered above. Studies of the surface modes in the system in question also will be published elsewhere.

We wish to thank A. M. Ermolaev for reading the manuscript and for valuable discussions.

1. E. A. Kaner and V. G. Skobov, Adv. Phys. 17, 605 (1968).

2. A. A. Abrikosov, Fundamentals of the Theory of Metals, North-Holland, Amsterdam (1988).

3. O. V. Konstantinov and V. I. Perel, Zh. Eksp. Teor. Fiz. 38, 161 (1960).
4. E. A. Kaner and V. G. Skobov, Zh. Eksp. Teor. Fiz. 45 610 (1963)

5. M. S. Khaikin, L. A. Falkovskii, V. S. Edelman, and R. T. Mina, Zh. Eksp. Teor. Fiz. 45, 1704 (1963).

6. E. A. Kaner and V. G. Skobov, Fiz. Tverd. Tela 6, 1104 (1964).

7. E. A. Kaner and N. M. Makarov, Zh. Eksp. Teor. Fiz. 58, $1972(1970)$.

8. E. A. Kaner and A. M. Ermolaev, Pis'ma Zh. Eksp. Teor. Fiz. 44, 391 (1986).

9. E. A. Kaner and A. M. Ermolaev, Zh. Eksp. Teor. Fiz. 92 , 2245 (1987)

10. F. G. Bass, A. A. Bulgakov, and A. P. Tetervov, High-frequency Properties of Semiconducting Superlattices, Nauka, Moskow (1989) (in Russian).

11. A. L. Fetter, Ann. Phys. 88, 1 (1974).

12. J. F. Giuliani and J. J. Quinn, Phys. Rev. Lett. 51, 919 (1983).

13. J. Yang and C. D. Gong, Phys. Lett. A128, 198 (1988).

14. V. M. Gvozdikov, Fiz. Nizk. Temp. 16, 1156 (1990).

15. W. Que and G. Kirczenov, Phys. Rev. B36, 6595 (1987).

16. R. D. King-Smith and J. Inkson, Phys. Rev. B36, 6501 (1987).

17. H. C. Oji and A. H. Mac Donald, Phys. Rev. Lett. 58, 824 (1987).

18. A. Griffin and A. J. Pindor, Phys. Rev. B39, 11503 (1989).

19. V. M. Gvozdikov, Physica C224, 293 (1994).

20. V. M. Gohfeld, M. I. Kaganov, and V. G. Peschansky, Fiz. Nizk. Temp. 12, 1173 (1986)

21. V. G. Peschansky, H. Kheir Bek, and S. N. Savel'eva, Fiz. Nizk. Temp. 18, 1012 (1992).

22. A. Tselis and J. J. Quinn, Phys. Rev. B29, 3318 (1984).

23. K. I. Golden and G. Kalman, Phys. Rev. B52, 14719 (1995).

24. L. Wendler and M. I. Kaganov, Phys. Status Solidi B142, K63 (1987).

25. B. N. Narahari Achar, Phys. Rev. B37, 10423 (1988).

26. I. D. Vagner and D. Bergman, Phys. Rev. B35, 9856 (1987).

27. The Quantum Hall Effect, R. E. Prange and S. Girvin (eds.), Springer-Verlag, N. Y. (1987).

28. F. Wilzek, Fractional Statistics and Anyon Superconductivity, World Scientific, Singapure (1990).

29. H. L. Stormer, J. P. Eisenstein, A. C. Gossard, W. Wiegmann, and K. Baldwin, Phys. Rev. Lett. 56, 85 (1986).

30. L. P. Gor'kov, Sov. Phys. Uspekhi 27, 809 (1984).

31. P. M. Chaikin, J. Phys. (Paris) I6, 1875 (1996).

32. I. M. Lifshitz, M. Ya. Azbel, and M. I. Kaganov, The Electron Theory of Metals, Nauka, Moscow (1973).

33. B. Rosenstein and I. D. Vagner, J. Phys.: Condens. Matter 2, 497 (1990).

34. V. G. Peschanskii, Zh. Eksp. Teor. Fiz. 114, 676 (1998) [JETP 87, 369 (1998)].

35. V. M. Gohfel'd and V. G. Peschanskii, Ukr. Fiz. Zh. 37, 1595 (1992)

36. T. Ando, A. B. Fowler, and F. Stern, Rev. Mod. Phys. 54 437 (1982)

37. J. Ruvalds, Supercond. Sci. Technol. 9, 905 (1996).

38. A. S. Rozhavsky, Synthetic Metals 70, 1019 (1995).

39. M. S. Kushwaha, Phys. Rev. B35, 3871 (1987). 